Recibido: $28 / 02 / 2016$

Aprobado versión definitiva: 18/04/2016

\title{
UNIFICACIÓN DE ORDENANZAS AMBIENTALES: SIERRA TECAPA CHINAMECA, HACIA LA SOSTENIBILIDAD LOCAL
}

\author{
RODOLFO MISAEL MEJÍA DIETRICH ${ }^{1}$
}

\section{RESUMEN}

La experiencia ha demostrado que la redacción de ordenanzas municipales ha estado presidida por dos principios fundamentales: la observancia del bien común y la potestad administrativa. La finalidad del estudio consistió en realizar una unificación de ordenanzas ambientales para la protección, conservación y recuperación de los recursos naturales en la Sierra Tecapa-Chinameca. Las unidades de análisis fueron: alcaldes y técnicos ambientales de las municipalidades de la Sierra. Siguiendo a Mertens (2005) \& Colmenares (2012), se adoptó la Investigación-Acción Participativa. El análisis fue cualitativo. Se encontró que existe conciencia de parte de los gobiernos locales sobre la necesidad de abordar el desarrollo sostenible y el cumplimiento de la legislación ambiental.

Palabras clave: Sierra Tecapa-Chinameca, recursos naturales, desarrollo sostenible, ordenanzas ambientales, El Salvador.

\footnotetext{
${ }^{1}$ Candidato a Magister en Métodos y Técnicas de Investigación Social de la Universidad de El Salvador. Afiliado a la Universidad de Oriente. 4ta. Calle Poniente No 705, Barrio San Felipe, San Miguel, El Salvador. Correo electrónico: rmejia@univo.edu.sv
} 


\title{
MOUNTAIN RANGE TECAPA-CHINAMECA: UNIFICATION OF ENVIRONMENTAL ORDINANCES TOWARD LOCAL SUSTAINABILITY
}

\begin{abstract}
Experience has shown that drafting municipal ordinances has been governed by two main principles: common good observance and administrative power. The purpose of this research was to make a unification of environmental ordinances for the protection, conservation and recovery of natural resources from Tecapa-Chinameca mountain range. The subjects of analysis were mayors and environmental technicians of the municipalities from the mountain range. Following Mertens (2005) \& Colmenares (2012), the type of study was Participatory Action Research. The analysis was qualitative. Finally, it was found that there is environmental concern of local governments on the need to address sustainable development and fulfillment of environmental legislation.
\end{abstract}

Key words: Tecapa-Chinameca mountain range, natural resources, sustainable development, environmental ordinances, El Salvador.

\section{Introducción}

La Asociación Intermunicipal de la Sierra Tecapa-Chinameca (ASITECHI), fundada el 27 de enero de 2011, aglutina los municipios de: Alegría, Jucuapa, Nueva Guadalupe, Chinameca y Santa Elena. Posteriormente, se incorporaron California, Santiago de María, Nueva Granada, Mercedes Umaña, El Triunfo, Berlín y Estanzuelas, todos están ubicados en los departamentos de San Miguel y Usulután.

Sin duda, la experiencia ha demostrado que la redacción de las ordenanzas locales ha estado presidida por dos principios fundamentales: a) la observancia del bien común: un concepto de elaboración clásica y patrística, el cual reconoce que el interés general debe estar por encima de los intereses particulares; y b) el de la potestad administrativa: aplicado como instrumento para conseguir la práctica del bien común. 
Hasta el presente, la Sierra Tecapa-Chinameca constituye la tercera zona en importancia de producción de café a escala nacional y es una reserva ecológica importante para la conservación de la biodiversidad y la provisión de servicios ambientales hacia la población (El Diario de Hoy, 2011).

En la actualidad las ordenanzas municipales de medio ambiente con la que cuentan algunos municipios de la Sierra Tecapa-Chinameca, necesitan una reforma estructural para vincularlas con todos los municipios de la Sierra. Esto con el fin de llenar un campo sectorial indispensable en la promoción de la calidad de vida de la población misma, fomentando las actividades productivas sostenibles, la sensibilización ambiental, la protección y puesta en valor del territorio, su biodiversidad, su riqueza cultural y etnográfica.

Bajo ese enfoque, el estudio sostuvo como interés principal la unificación de ordenanzas municipales ambientales para la protección, conservación y recuperación de los recursos naturales en la Sierra Tecapa-Chinameca. De igual manera, dinamizar el intercambio de experiencias entre municipalidades de la Sierra para el crecimiento y mejora de la normativa ambiental local.

Por otra parte, la falta de regulación legal de los recursos naturales por parte de los gobiernos locales que conforman la Sierra Tecapa-Chinameca a excepción de la Ley del Medio Ambiente (1998), y la poca normativa de ordenanzas ambientales obsoletas y no concordantes con la realidad nacional que se vive en la Sierra, produce la carencia de un instrumento legal que regule lo conexo a recursos naturales, lo cual tiene implicación directa en el orden económico y social que enmarcan los pilares del desarrollo y sostenibilidad local.

\section{Planteamiento del Problema}

Tomando en cuenta, que El Salvador es de pequeña extensión territorial, con sobre población, con pocos recursos naturales, los cien municipios asentados en bosques de café, representan el $75 \%$ del bosque nacional, cuyos valores económicos, los servicios ambientales renovables, son una muestra significativa para elaborar la teoría de sostenibilidad local (Sancho, 2011). 
Por otra parte, en varios municipios del país se está experimentando la búsqueda de alternativas económicas generalmente no tradicionales, que rompan la inmovilidad de la vida económica local con pequeños resultados inmediatos. Además, constituyan hechos demostrativos y detonantes para crear bases de una economía alternativa que sustituya, reactive o complemente las actividades que en las últimas dos décadas han dejado de ser productivas para el país (SACDEL y CND, 2004).

En virtud de lo anterior, la ASITECHI tiene como propósitos prioritarios: conducir estratégicamente el desarrollo integral y sostenible de la Sierra como un solo territorio, así como mejorar la calidad de vida de sus habitantes, promoviendo los principios de solidaridad, equidad, transparencia, pluralismo y participación ciudadana. Una buena gestión de los recursos naturales no es perjudicial para el desarrollo, así para algunos, la ordenación del medio ambiente es un complemento del desarrollo (Silva, Macarrein, \& Forclaz, 2002).

Los municipios de la Sierra poseen valores y caracteres históricos vinculados como administración más próxima a los ciudadanos. Se ha modelado desde el pasado un marco normativo basado en la experiencia de la convivencia ciudadana, lo que ha marcado en la historia periodos de progreso social, mediante la progresiva y creciente participación de los vecinos. Los municipios se han convertido en una unidad administrativa, jurídica, activa y dinámica.

Tomando como base estas potencialidades del territorio, se ha pensado en la unificación de las ordenanzas municipales ambientales. Ello con el fin de generar municipios más competitivos, amigables con los negocios y el medio ambiente, atractivos para las inversiones lo que significará sin lugar a dudas un avance hacia la sostenibilidad local y contribuir al desarrollo sostenible de la Sierra. Para Díaz Palacios (2005) el desarrollo de una localidad está vinculado con sus recursos, capacidades, potencialidades y con el desarrollo del país. 


\section{Estado del arte}

\subsection{Marco histórico}

La República de El Salvador posee una extensión territorial de $21.040 \mathrm{~km}^{2}$, a la fecha y según la encuesta de Hogares y Propósitos Múltiples (EHPM), cuenta con una población total de 6.401 .415 personas. De estos, 3.989.266 residen en el área urbana y 2.412.149 en la rural, lo que en términos relativos representa el $62.3 \%$ y $37.7 \%$, respectivamente (DIGESTYC, 2015). Administrativamente, se divide en 14 departamentos y 262 municipios (Sáenz, 2010).

El Salvador era hasta hace poco un país predominantemente agropecuario; sin embargo, el agro ha ido perdiendo peso como generador de divisas y ha cedido su puesto a otras actividades económicas como las maquilas y las remesas. Esta dinámica, junto a las migraciones internas e internacionales, están generando importantes transformaciones territoriales, que al realizarse al margen de una planificación estructurada, impactan negativamente en las distintas zonas del país, tanto urbanas como rurales, profundizando los desequilibrios territoriales (COMURES, 2008). Por otro lado, los procesos de degradación ambiental se han incrementado en El Salvador debido a una combinación de causas naturales, antropogénicas y del cambio climático (Sáenz, 2010).

Figura 1. Mapa de El Salvador y ubicación zona de estudio

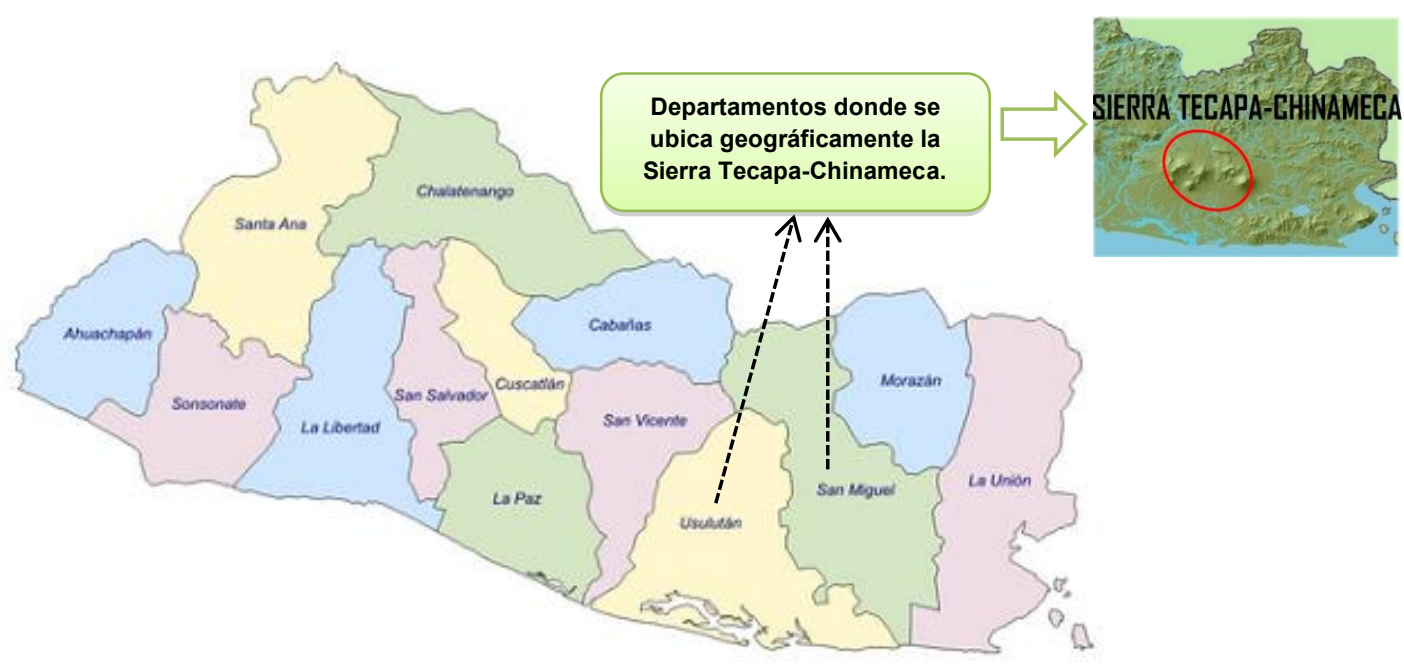

Fuente: Elaboración propia 
La Sierra Tecapa-Chinameca es una cadena de cerros, volcanes y montañas y se eleva desde la margen oriental del río Lempa y finaliza en el curso medio del río Grande de San Miguel. Sus elevaciones orográficas más notables son los cerros Taburete, Tigre, Oromontique y los volcanes de Tecapa, Usulután y Jucuapa en el departamento de Usulután y el volcán Chaparrastique en el departamento de San Miguel. Su ubicación geográfica corresponde a los departamentos de San Miguel y Usulután (FUNDE. Fundación Nacional para el Desarrollo, 2011).

A la fecha se vienen apoyando varias iniciativas en el desarrollo territorial de la Sierra Tecapa-Chinameca, las cuales se encuentran en diferentes fases. E propósito fundamental es contribuir a crear un dinamismo nuevo, necesario y alternativo para el desarrollo ambiental y económico de la Sierra. Todo lo anterior, en armonía con el Plan Quinquenal de Desarrollo 2014-2019 implementado por la Presidencia de la República.

\subsection{Marco jurídico}

\subsubsection{Constitución de la República de El Salvador}

La Constitución de la República de El Salvador en su artículo 117, dispone que es deber del Estado proteger los recursos naturales, así como la diversidad e integridad del medio ambiente, para garantizar el desarrollo sostenible y declara de interés social la protección, conservación, aprovechamiento racional y restauración de los recursos naturales. Por ello, mediante Decreto Ejecutivo No. 27 del 16 de mayo de 1997, se crea el Ministerio de Medio Ambiente y Recursos Naturales. Las competencias de esta Secretaría de Estado se establecen en el Reglamento Interno del Órgano Ejecutivo, Decreto No. 30 emitido por el Consejo de Ministros el 19 de mayo de 1997 (MARN, 2006). 


\subsubsection{Código Municipal}

La base legal para la creación de ordenanzas municipales emana de lo estipulado en el Código Municipal, que es aplicable a los 262 Municipios de El Salvador. Por tal motivo, el Código en su artículo 3 numeral $5^{\circ}$ establece: "que la autonomía del municipio se extiende al decreto de ordenanzas y reglamentos locales".

\subsubsection{Ley del Medio Ambiente}

En 1998 se emite la Ley del Medio Ambiente, con el objeto de desarrollar las disposiciones que tiene la Constitución de la República, relativas a la protección, conservación y recuperación del medio ambiente. También, para la formación de la gestión ambiental pública y privada, y asegurar la aplicación de los tratados o convenios internacionales celebrados por El Salvador. Esta Ley fue promulgada por Decreto Legislativo No. 223 del 2 de marzo de 1998, publicado en el Diario Oficial No. 79, tomo 339 del 4 de mayo de 1998 (MARN, 2006).

Posteriormente, con el propósito de desarrollar las normas y preceptos de la Ley del Medio Ambiente, se emitió el Reglamento General, el cual se convierte en su instrumento ejecutorio principal. Este reglamento fue aprobado a través del Decreto Ejecutivo No. 17 de mayo del año 2000 y publicado en el Diario Oficial No. 73, tomo 347 del 12 de abril de 2000.

Para dar cumplimiento al artículo 114 de la Ley del Medio Ambiente, se emitieron los Decretos Ejecutivos No. 38, 39, 40 y 42 de fecha 31 de mayo de 2000. Los reglamentos especiales sobre el Control de las Sustancias Agotadoras de la Capa de Ozono; de Aguas Residuales; Normas Técnicas de Calidad Ambiental; en Materia de Sustancias, Residuos y Desechos Peligrosos; sobre el Manejo Integral de los Desechos Sólidos fueron publicados en el Diario Oficial No. 101, tomo 347 de fecha 01 de junio de 2000. El Reglamento Especial para la Compensación Ambiental por Decreto Ejecutivo No. 50, del 05 de mayo de 2004 fue publicado en el Diario Oficial No. 93, tomo 363 de fecha 21 de mayo de 2004 (MARN, 2015). 


\subsubsection{Política Nacional de Medio Ambiente}

El Gobierno de El Salvador, mediante la Política Nacional del Medio Ambiente (2012) reconoce que los recursos naturales y el medio ambiente vinculan a todos los sectores del país, e involucra a diversas instituciones públicas y privadas, centralizadas y descentralizadas. Por ello mismo, es necesario orientar y ordenar la gestión por un ambiente ecológicamente equilibrado y en constante ajuste a las necesidades humanas dentro de un marco sostenible.

La Política, comprende dos áreas temáticas: a) Conservación y aprovechamiento de los recursos naturales, en la que se incluye: el ordenamiento territorial, agua, aire, suelo, biodiversidad y bosque; b) La gestión ambiental que es consistente con el marco jurídico e institucional, educación ambiental, participación social, marco económico, enfoque de equidad de género y prevención y control de la contaminación.

\subsection{Situación ambiental El Salvador}

Según informes del Ministerio del Medio Ambiente y Recursos Naturales (MARN), en el país estamos deforestando un promedio anual de 4.500 hectáreas de bosques y hemos reducido las áreas naturales a $1.87 \%$ en todo el territorio nacional (Alvarado, 2006). Los recursos hídricos de El Salvador se estiman en $17.8 \mathrm{~km}^{3}$, de los cuales 11.6 provienen de las aguas superficiales. El $84 \%$ de esta escorrentía superficial ocurre durante la estación lluviosa y $16 \%$ durante la estación seca. El Salvador es el único país de Centro América cuyo territorio drena enteramente hacia la vertiente del Océano Pacífico. Existen aportes de aguas superficiales provenientes de Honduras y Guatemala, aproximadamente de 7.5 $\mathrm{km}^{3}$ por año, por lo que los recursos hídricos totales se estiman en $25.2 \mathrm{~km}^{3}$ por año (MARN, 2006).

Los 360 ríos de todo el país, en los últimos 30 años han sufrido un impacto de disminución de su caudal que va de un $30 \%$ a un $70 \%$ y en el norte de Cuscatlán, Chalatenango y Cabañas, de un $70 \%$ a un $100 \%$, en donde ya existen 
zonas desérticas. El único afluente que ha incrementado su caudal de un $25 \%$ a un $35 \%$ es el río Acelhuate (Alvarado, 2006).

La Política Nacional del Medio Ambiente (2012) reconoce que la problemática central a la que debe dirigirse es la severa degradación ambiental y vulnerabilidad creciente del país frente al cambio climático. Esa problemática central se expresa en los siguientes problemas específicos: a) degradación de ecosistemas de gran valor; b) insalubridad ambiental generalizada; c) crítico estado del recurso hídrico; d) desordenada ocupación del territorio; e) amenaza climática creciente; f) escasa cultura de responsabilidad y cumplimiento ambiental.

\subsection{Fundamentos de la sostenibilidad y desarrollo sostenible}

Con el transcurso del tiempo, los conceptos de sostenibilidad y desarrollo sostenible se han hecho populares en los medios de comunicación a raíz del documento titulado "Nuestro futuro común", que fue elaborado en 1987 por la entonces Primer Ministra de Noruega, Gro Harlem Brundtland. Por tal razón, también se le conoce con el nombre de Informe de la Comisión Brundtland. En ese documento se define como sostenible "aquel desarrollo que satisface las necesidades del presente sin comprometer la capacidad de las generaciones futuras para satisfacer sus propias necesidades" (UNESCO, 2012). La anterior definición, fue pieza clave y fundamental para la génesis de la filosofía del desarrollo sostenible (Gómez de Segura, 2014).

Por lo tanto, la sostenibilidad es un paradigma para pensar en un futuro en el cual las consideraciones ambientales, sociales y económicas se equilibran en la búsqueda del desarrollo y de una mejor calidad de vida. Estos tres ámbitos fundamentales -sociedad, medio ambiente y economía- están entrelazados. Por ejemplo, una sociedad próspera depende de un medio ambiente sano que provea de alimentos y recursos, agua potable y aire limpio a sus ciudadanos.

En el origen del concepto de sostenibilidad existen dos cosas primordiales: a) la percepción de la gravedad de los desequilibrios medioambientales, observados en diferentes lugares del mundo; b) la conciencia de la posibilidad de 
una crisis ecológica global con consecuencias imprevisibles (pero previsiblemente catastróficas) para el futuro de nuestro planeta y de la mayoría de las especies que habitan en él. La idea de sostenibilidad empezó siendo, pues, una respuesta preventiva ante la perspectiva de colapso global o parcial del modelo de civilización dominante (Riechmann, 2006).

En relación al concepto de desarrollo sostenible también llamado "desarrollo sustentable" o "perdurable", después de su marco filosófico expuesto en el Informe Brundtland (1987) cuyo resultado fue producto de los trabajos de la Comisión Mundial del Medio Ambiente y Desarrollo de la Organización de las Naciones Unidas (ONU). En esa línea, Juncos-Gautier (2012) lo define como "posibilidad de prolongar la productividad del uso de los recursos naturales a lo largo del tiempo, a la vez que se mantiene la integridad de esos recursos, viabilizando la continuidad de su uso para las próximas generaciones".

De igual manera, la Declaración de Río sobre el Medio Ambiente y el Desarrollo, conocida con el nombre de "Cumbre de la Tierra", sostuvo que "el derecho al desarrollo debe ejercerse en forma tal que responda equitativamente a las necesidades de desarrollo y ambientales de las generaciones presentes y futuras" (Principio 3 Declaración de Rio, 1992).

Bajo este contexto, podríamos preguntarnos, ¿cuál es la diferencia entre sostenibilidad y desarrollo sostenible? La sostenibilidad suele considerarse como un objetivo a largo plazo (es decir, un mundo más sostenible), mientras que el desarrollo sostenible se refiere a los muchos procesos y caminos que existen para lograr ese objetivo. Por ejemplo, la agricultura y silvicultura sostenible, la producción y consumo sostenible, el buen gobierno, la investigación y transferencia tecnológica, la educación y formación, entre otros.

En definitiva, un nivel de sostenibilidad es necesario para enfrentarse a los retos globales en un mundo de incertidumbre y complejidad -la sierra TecapaChinameca, no es la excepción-. La sostenibilidad debe ser un elemento fundamental en la estrategia de los gobiernos locales que busquen crecimiento en todos sus niveles. El objetivo del desarrollo sostenible es lograr un equilibrio entre 
las dimensiones sociales, económicas y ambientales, en es una escala local, nacional, regional y global (Consejo de Liderazgo RSDS, 2013).

\subsection{Generalidades del análisis FODA}

Las siglas provienen del acrónimo en inglés SWOT (strenghts, weaknesses, opportunities, threats); en español, aluden a fortalezas, oportunidades, debilidades y amenazas. El análisis FODA o DOFA consiste en realizar una evaluación de los factores fuertes y débiles que, en su conjunto, diagnostican la situación interna de una organización, así como su evaluación externa, es decir, las oportunidades y amenazas. También, es una herramienta que puede considerarse sencilla y que permite obtener una perspectiva general de la situación estratégica de una organización determinada (Ponce Talancón, 2007).

Por otra parte, la exploración de los factores positivos y negativos, internos y externos que tienen efectos sobre la institución permitirá elaborar un estudio estratégico en el que se identifiquen las fortalezas, oportunidades, debilidades y amenazas de la entidad (SENPLADES, 2012). En efecto, el objetivo principal de este análisis es potenciar las fortalezas de la organización para aprovechar oportunidades, contrarrestar amenazas y corregir debilidades (García López \& Cano Flores, 2013).

Para el análisis FODA, una vez identificados los aspectos fuertes y débiles de una organización se debe proceder a la evaluación de ambos. Es importante destacar que algunos factores tienen mayor preponderancia que otros: mientras que los aspectos considerados fuertes de una organización son los activos competitivos, los débiles son los pasivos también competitivos. El éxito de la dirección es diseñar estrategias a partir de las que la organización realiza de la mejor manera, obviamente tratando de evitar las estrategias cuya probabilidad de éxito se encuentre en función de los pasivos competitivos (Wilhelm, 1992).

En síntesis, este análisis permite decidir sobre el futuro, ayuda a plantear las acciones que deberíamos poner en marcha para aprovechar las oportunidades detectadas y a preparar a nuestra organización contra las amenazas teniendo 
conciencia de nuestras debilidades y fortalezas. Además, constituye una gran importancia dentro de la dirección estratégica (Rodríguez, 2012).

\section{Metodología}

\subsection{Tipo de investigación}

La investigación fue de corte cualitativo con un diseño investigación-acción participativa (IAP), que involucra a los miembros del grupo o comunidad en todo el proceso del estudio (desde el planteamiento del problema hasta la elaboración del reporte) y la implementación de acciones, producto de la indagación (Mertens, 2005 y Colmenares, 2012).

\subsection{Población y muestra}

La población que fue objeto de estudio comprendió, los alcaldes y técnicos de las Unidades de Medio Ambiente de las municipalidades de la Sierra que conforman la Asociación Intermunicipal de la Sierra Tecapa-Chinameca (ASITECHI). En la presente investigación se seleccionó la muestra denominada muestras homogéneas, en que las unidades seleccionadas poseían un mismo perfil con características similares (Hernández Sampieri, Fernández Collado, \& Baptista Lucio, 2010). La selección de los entrevistados se determinó a partir de criterios de calidad e idoneidad, así como de su experiencia en materia ambiental (Ver tabla 1).

\section{Tabla 1. Estratificación de la muestra}

\begin{tabular}{lc}
\hline Población & Cantidad \\
\hline Alcaldes municipales (ASITECHI) & 12 \\
Técnicos ambientales & 12 \\
\hline Total & $\mathbf{2 4}$ \\
\hline
\end{tabular}

Fuente: Elaboración propia

\subsection{Técnicas e instrumentos de recolección de información}

Se utilizaron las técnicas clásicas de la metodología cualitativa, como lo son la observación participativa, sesiones en profundidad o grupos de enfoque y la entrevista estructurada o dirigida. En esta última nos apoyamos de medios 
audiovisuales (videocámara y grabadora de voz) y el instrumento utilizado fue el cuestionario. De igual manera, la revisión bibliográfica y documental de información relacionada con el desarrollo sostenible.

El propósito de la entrevista de investigación cualitativa es obtener descripciones del mundo vivido por las personas entrevistadas, con el fin de lograr interpretaciones fidedignas del significado que tienen los fenómenos descritos (Kvale, 1996).

\subsection{Técnicas de análisis}

Para el tratamiento de la información obtenida mediante la entrevista dirigida, se utilizó el análisis cualitativo, que se llevó a cabo a través de una matriz, la cual se dividió en categorías de análisis previamente definidas como prioritarias para la comprensión y profundización de diversas dimensiones de la situación ambiental en la Sierra Tecapa-Chinameca.

\section{Análisis de resultados}

\subsection{Matriz FODA}

El estudio sobre la normativa ambiental local en materia de medio ambiente de los municipios de la Sierra nos conduce a unas primeras conclusiones tratadas con análisis FODA. Las fortalezas y oportunidades definen los puntos fuertes del proceso con idéntica proyección, debilidades y amenazas constituyen los puntos débiles del proceso analizado desde una proyección sincrónica. 
Tabla 2. Resumen FODA: Unificación de ordenanzas ambientales

\begin{tabular}{|c|c|c|}
\hline & Fortalezas (F) & Debilidades (D) \\
\hline $\begin{array}{l}\text { Factores } \\
\text { Externos }\end{array}$ & $\begin{array}{l}\text { 1. Creación de Unidades de Medio } \\
\text { Ambiente. } \\
\text { 2. } 100 \% \text { de los Alcaldes apoya el } \\
\text { desarrollo sostenible. } \\
\text { 3. Programas de capacitación para } \\
\text { técnicos. } \\
\text { 4. Vinculación con entidades de } \\
\text { protección ambiental. }\end{array}$ & $\begin{array}{l}\text { 1. Falta de recursos económicos } \\
\text { para Unidades de Medio Ambiente. } \\
\text { 2. Falta de apoyo para financiar } \\
\text { proyectos ambientales. } \\
\text { 3. Inestabilidad laboral técnicos } \\
\text { ambientales. }\end{array}$ \\
\hline Oportunidades (0) & FO (Maxi-Maxi) & DO (Mini-Maxi) \\
\hline $\begin{array}{l}\text { 1. Realizar diagnósticos ambientales y } \\
\text { planes de acción. } \\
\text { 2. Formular políticas públicas } \\
\text { centrales con la variable ambiental. } \\
\text { 3. Destinar subvenciones para la } \\
\text { mejora ambiental. } \\
\text { 4. Crear bases de datos con acceso a } \\
\text { normativa ambiental actualizada. } \\
\text { 5. Gestionar modelos regionales de } \\
\text { creación de Unidad de Gestión } \\
\text { Ambiental. }\end{array}$ & $\begin{array}{l}\text { 1. Desarrollar perfiles de proyectos } \\
\text { ambientales para buscar la gestión } \\
\text { de fondos con cooperantes } \\
\text { nacionales e internacionales (O1, } \\
\text { F1) } \\
\text { 2. Extender la promoción de } \\
\text { políticas públicas con enfoque } \\
\text { verde-protección ambiental- (O2, } \\
\text { F2) } \\
\text { 3. Fortalecer los programas de } \\
\text { capacitación ambiental }(\mathrm{O} 3, \mathrm{O} 4, \mathrm{O} 5 \text {, } \\
\text { F3, F4) }\end{array}$ & $\begin{array}{l}\text { 1. Gestionar la búsqueda de fondos } \\
\text { para financiar proyectos } \\
\text { ambientales de especial interés para } \\
\text { la Sierra }(\mathrm{O} 1, \mathrm{O} 2, \mathrm{O} 3, \mathrm{D} 1, \mathrm{D} 2) \\
\text { 2. Crear programa de incentivos } \\
\text { para promover la estabilidad laboral } \\
\text { (O2, D3) }\end{array}$ \\
\hline Amenazas (A) & FA (Maxi-Mini) & DA (Mini-Mini) \\
\hline $\begin{array}{l}\text { 1. Débil participación ciudadana en } \\
\text { políticas públicas ambientales. } \\
\text { 2. Débiles programas educativos en } \\
\text { educación ambiental. } \\
\text { 3. Desinterés ciudadano por contar } \\
\text { con un marco normativo ambiental. }\end{array}$ & $\begin{array}{l}\text { 1. Establecer cabildos abiertos para } \\
\text { escuchar el pensar de la población } \\
\text { (A1, F2) } \\
\text { 2. Promover programas de } \\
\text { educación ambiental para la } \\
\text { sociedad civil (A2, A3, F1, F2, F4) }\end{array}$ & $\begin{array}{l}\text { 1. Revisión del Plan Operativo Anual } \\
\text { de acuerdo a las necesidades de la } \\
\text { población con posibilidad de adoptar } \\
\text { un plan de ajuste de presupuesto } \\
\text { para rubros ambientales (A1, A2, } \\
\text { A3, D1, D2) }\end{array}$ \\
\hline
\end{tabular}

Fuente: Elaboración propia en base a talleres con Alcaldes y Técnicos Ambientales

En lo referente a las fortalezas, son características internas del sistema que favorecen o suponen ventajas para que pueda satisfacer las necesidades y expectativas de la población (capacidades y recursos) por lo tanto, contribuyen a una buena calidad de vida de todos los vecinos. La existencia de estos puntos fuertes dentro de la Sierra, facilita el cumplimiento de la normativa ambiental y su eficacia. Por ello, no sólo hay que preservar dichas fortalezas, sino que los gobiernos locales deben trabajar para aumentarlas. En lo vinculante a las oportunidades, son circunstancias coyunturales, generalmente externas, que 
pueden influir favorablemente en el funcionamiento del sistema y de las que puede beneficiarse. Ejemplo: apoyo logístico y técnico del Ministerio de Medio Ambiente y Recursos Naturales para la implantación de las ordenanzas ambientales en la Sierra.

En lo concerniente a las debilidades, son las características internas del sistema que impiden que pueda satisfacer, por sí mismo, las necesidades y expectativas del proceso de unificación de ordenanzas ambientales. La política de la administración municipal no debe centrarse únicamente en atacar las debilidades existentes sino en la toma de medidas necesarias para evitar la aparición de nuevas debilidades que puedan afectar negativamente a la protección del medio ambiente. Finalmente, tenemos a las amenazas, se describen como circunstancias coyunturales, generalmente externas, que pueden influir negativamente en el funcionamiento del sistema. Ejemplo: Desinterés ciudadano por contar con un marco normativo ambiental.

En resumen, las debilidades y fortalezas son propiedades o características internas del sistema, mientras que las amenazas y oportunidades son generalmente externas a él. No obstante, las fortalezas encontradas en las municipalidades de la Sierra: gobiernos locales totalmente comprometidos con el desarrollo sostenible y técnicos ambientales, altamente capacitados en el área ambiental; a contrario sensu, existe una escasa participación ciudadana en temas ambientales, situación preocupante para los alcaldes, porque limita la formulación de políticas públicas locales con enfoque verde.

\subsection{Sostenibilidad local en la Sierra Tecapa-Chinameca}

Existe en la Sierra, suficiente capital técnico, humano y natural para asegurar un futuro medianamente confortable a toda su población de 166.233 habitantes (DIGESTYC, 2015). La protección de sus recursos naturales, busca el impulso de proyectos relacionados con el potencial turístico que posee para su desarrollo y sostenibilidad local, con la finalidad de explotar ese potencial en completa armonía con el medio ambiente. 


\section{Figura 2. Componentes de la sostenibilidad local en la Sierra}

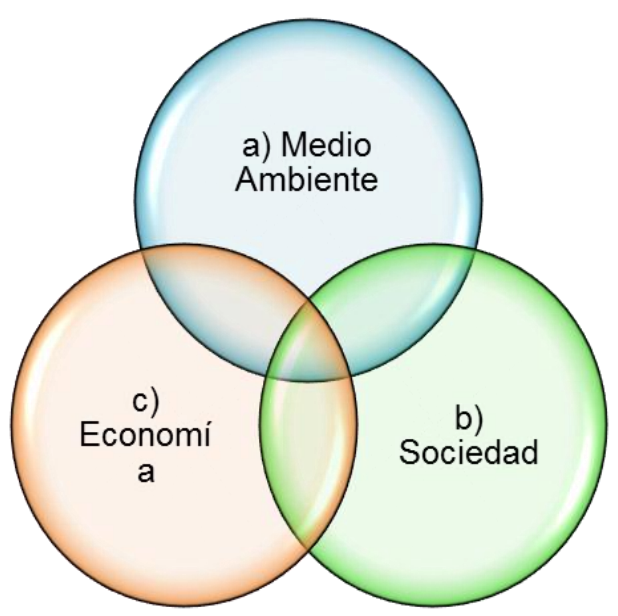

Fuente: Elaboración propia en base a los componentes del Desarrollo Sostenible

En primer lugar, el medio ambiente, tiene un avance transcendental en la Sierra: existe un bajo nivel de urbanización, todos los municipios tienen servicio de recolección de desechos sólidos y gobiernos locales comprometidos con el desarrollo sostenible. También existen iniciativas para el manejo de desechos sólidos orgánicos en algunos municipios, unidades de medio ambiente que velan por la protección ambiental; hay recursos renovables en abundancia y promoción de turismo sustentable pero hace falta un elemento esencial, la gobernanza ambiental actualizada. Por lo tanto, no podemos hablar de una sostenibilidad local primordial en términos de medio ambiente, se está haciendo el esfuerzo necesario, pero tales acciones no son suficientes, ejemplo: educación ambiental sigue siendo tarea pendiente en la Sierra.

Por último y no menos importante, el componente económico, el índice de competitividad municipal ha mejorado en los últimos dos años para todas las municipalidades de la Sierra: la seguridad ciudadana, carga regulatoria, tasas e impuestos, transparencia, pagos informales entre otros. Pero el mayor problema para abordar el tema de la sostenibilidad, es el desempleo. Existen niveles moderados de desempleo (DIGESTYC, 2015), situación que limita en gran medida 
el avance hacia la sostenibilidad local. Una salida alternativa la ha constituido la generación de emprendimiento en turismo sustentable.

En síntesis, la educación ambiental juega un papel importante como catalizador del desarrollo hacia la sostenibilidad local de la Sierra. Se necesita educación ambiental para desarrollar valores que promuevan la conservación y el sabio uso de recursos naturales a la vez que se construye una sociedad donde la distribución de estos recursos y el impacto de las actividades productivas sean más equitativos. Para caminar hacia esa dirección es necesario educar sobre la necesidad de la reevaluación constante de metas y objetivos del desarrollo local como proceso integral.

\section{Discusión y valoración de los hallazgos}

Con análisis FODA se obtuvieron conclusiones útiles para la identificación de objetivos y definición de las líneas de acción desde los planteamientos del proyecto de unificación de ordenanzas ambientales para la Sierra TecapaChinameca. La finalidad del estudio fue buscar la protección, conservación y recuperación de los recursos naturales de la Sierra Tecapa-Chinameca como un marco normativo ambiental que sirva de plataforma de avance hacia la sostenibilidad local de la Sierra.

En talleres y conversatorios con alcaldes y técnicos de las unidades ambientales de la Sierra, se recogieron los datos y se analizaron con métodos cualitativos, según los resultados se cumplieron los objetivos del estudio. También, se presentó a los alcaldes de la Sierra, un anteproyecto de unificación de ordenanzas municipales ambientales con ámbito de aplicación exclusiva para toda la Sierra.

Además con el intercambio de experiencias en materia ambiental entre todas las municipalidades de la Sierra, se respondió a los enunciados de la investigación, dado el caso que sin objeción alguna los intercambios de experiencias contribuyen positivamente a la mejora de la normativa ambiental local. Asimismo, con la unificación de ordenanzas ambientales se tendrá un mejor 
aprovechamiento de los recursos naturales de la Sierra, prueba de esta afirmación es la creación de la UGAMA (Unidad de Gestión Ambiental de la ASITECHI), se sabe que una buena gestión de los recursos naturales no es perjudicial para el desarrollo, así para algunos, la ordenación del medio ambiente es un complemento del desarrollo (Silva, Macarrein, \& Forclaz, 2002).

\section{Conclusiones}

A la luz de los datos recolectados y al finalizar el estudio, se tienen las siguientes conclusiones:

* La Sierra Tecapa-Chinameca posee una diversidad de recursos naturales los cuales requieren una protección, conservación y recuperación a través de un instrumento jurídico que garantice un verdadero desarrollo sustentable considerando de manera integral aspectos sociales, económicos y ambientales que promuevan la sostenibilidad local.

* La participación ciudadana e inclusión social son fundamentales para lograr los procesos de gobernanza encaminados al desarrollo sustentable de la Sierra Tecapa-Chinameca, y contribuir además al fomento del derecho humano denominado derecho a un ambiente sano.

* El desarrollo de programas de formación para los integrantes de las Unidades de Medio Ambiente de las municipalidades de la Sierra TecapaChinameca juegan un papel fundamental en la aplicación de los instrumentos jurídicos que regulan los recursos naturales de la Sierra.

* Las debilidades en los procesos de gestión municipal, en relación al medio ambiente, instituyen que las municipalidades de la Sierra TecapaChinameca tienen que establecer formas en que los indicadores de desarrollo sustentable puedan ser incorporados en sus procesos de gestión para un avance hacia la sostenibilidad local de la Sierra.

* La unificación per se es suficiente para promover las actividades productivas; el nuevo cuerpo jurídico alcanzado, tiene entre sus funciones: la promoción de la calidad de vida de sus pobladores, el fomento de la 
sensibilización ambiental, la protección y puesta en valor del territorio, la biodiversidad, la riqueza cultural y etnográfica.

\section{Referencias bibliográficas}

Alvarado, M. (03 de 10 de 2006). Diagnóstico de la situación medio ambiental de El Salvador. Recuperado el 25 de 02 de 2013, de:

HYPERLINK

"http://www.ecoportal.net/Temas_Especiales/Desarrollo_Sustentable/Diagnostico_ de_la_situacion_medio_ambiental_de_El_Salvador"

http://www.ecoportal.net/Temas Especiales/Desarrollo Sustentable/Diagnostico d e la situacion medio ambiental de El Salvador

Asamblea Constituyente. El Salvador. (15 de Diciembre de 1983). Recuperado el 25 de Mayo de 2014, de Constitución: República de El Salvador:

HYPERLINK

"http://www.asamblea.gob.sv/eparlamento/indice-

legislativo/buscador-de-documentos-legislativos/constitucion-de-la-republica"

http://www.asamblea.gob.sv/eparlamento/indice-legislativo/buscador-de-

documentos-legislativos/constitucion-de-la-republica

Asamblea Legislativa. El Salvador. (05 de Febrero de 1986). Recuperado el 28 de Junio de 2014, de Código Municiapal:

HYPERLINK

"http://www.asamblea.gob.sv/eparlamento/indicelegislativo/buscador-de-documentos-legislativos/codigo-municipal"

http://www.asamblea.gob.sv/eparlamento/indice-legislativo/buscador-dedocumentos-legislativos/codigo-municipal

Asamblea Legislativa. El Salvador. (04 de Mayo de 1998). Recuperado el 23 de 04 de 2015, de Ley del Medio Ambiente:

HYPERLINK

"http://www.asamblea.gob.sv/eparlamento/indicelegislativo/buscador-de-documentos-legislativos/ley-del-medio-ambiente" http://www.asamblea.gob.sv/eparlamento/indice-legislativo/buscador-dedocumentos-legislativos/ley-del-medio-ambiente Colmenares, A. (2012). Investigación-Acción Participativa: Una metodología integradora del conocimiento y la acción. Voces y Silencio: Revista Latinoaméricana de Investigación, 3(1), 103-104.

COMURES. Corporación de Municipalidades de la República de El Salvador. (2008). Ordenamiento Territorial y Gestión Ambiental. Cuadernillo de Formación Municipal. San Salvador, El Salvador.

Consejo de Liderazgo de la Red de Soluciones para el Desarrollo Sostenible. (2013). Una agenda de acción para el Desarrollo Sostenible. New York: UNSDSN. Consejo de Ministros. (2012). Política Nacional de Medio Ambiente. San Salvador. 
Díaz Palacios, J. (2005). Manual del buen gobierno local: Enfoques, principios y estrategias. Lima: SINCO Editores.

DIGESTYC. Dirección General de Estadísticas y Censos. (2015). Encuesta de Hogares de Propósitos Multiples (EHPM) El Salvador 2014. San Salvador, El Salvador: Ministerio de Economía.

El Diario de Hoy. (06 de Diciembre de 2011). Recuperado el 09 de Marzo de 2015, de USAID apoya 50 Alcadias a ser competitivas:

HYPERLINK

"http://www.elsalvador.com/mwedh/nota/nota_completa.asp?idCat=47862\&idArt=6 446329"

http://www.elsalvador.com/mwedh/nota/nota completa.asp?idCat=47862\&idArt=64 46329

FUNDE. Fundación Nacional para el Desarrollo. (15 de Marzo de 2011). Una primera aproximación desde los territorios de Los Nonualcos, la Sierra Tecapa Chinameca y la Región del Valle Rio de Jiboa. San Salvador: FUNDE.

García López, T., \& Cano Flores, M. (2013). El FODA: Una técnica para el análisis de problemas en el contexto de la planeación en las organizaciones. Veracruz: Universidad Veracruzana.

García, E., \& Lozano Cutanda, B. (2006). Diccionario de Derecho Ambiental. Madrid: lustel, 1a. Edición.

Gómez de Segura, R. (2014). Del Desarrollo Sostenible según Brundtland a la sostenibilidad como biomimesis. Bilbao: Universidad del País Vasco.

Henry, H. (1980). Appraising a company's strengths and weaknesses. Managerial Plannig, 76-81.

Hernández Sampieri, R., Fernández Collado, C., \& Baptista Lucio, P. (2010). Metodología de la Investigación. México: Mc Graw Hill, 5a.Ed.

Juncos-Gautier, M. A. (2012). Sobre el Desarrollo Sustentable. Bayamón: Universidad Metropolitana.

Kvale, S. (1996). Interviews: An introduction to qualitave research interviewing. CA, EE.UU.: Sage.

MARN (a) Ministerio de Medio Ambiente y Recursos Naturales. (2006). Informe del Estado de Medio Ambiente de El Salvador año 2005. San Salvador: MARN.

MARN (b). Ministerio de Medio Ambiente y Recursos Naturales. (2015). Informe del Estado de Medio Ambiente de El Salvador año 2014. San Salvador: Ministerio de Medio Ambiente y Recursos Naturales.

Mertens, D. (2005). Research and evaluation in education and psychology: Integrating diversity with quantitative, qualitative, and mixed methods. CA, EE.UU.: Thousand Oaks, 2da.Ed. 
Moreno Pérez, S. (2007). El debate sobre el desarrollo sustentable o sostenible y las experiencias internacionales de desarrollo urbano sustentable. México D.F: Centro de Estudios Sociales y de Opinión Pública.

ONU. Organización de las Naciones Unidas. (2015). Declaración de Rio sobre el Medio Ambiente y el Desarrollo (1992). Recuperado el 03 de Junio de 2015, de HYPERLINK "http://www.un.org/spanish/esa/sustdev/agenda21/riodeclaration.htm" http://www.un.org/spanish/esa/sustdev/agenda21/riodeclaration.htm

Organo Ejecutivo. El Salvador. (12 de Abil de 2000).Recuperado el 28 de Junio de 2014, de Reglamento General de la Ley del Medio Ambiente:

HYPERLINK

"http://elsalvador.eregulations.org/media/reglamento\%20general\%20de $\% 201 a \% 20$ I ey\%20del\%20medio\%20ambiente.pdf"

http://elsalvador.eregulations.org/media/reglamento $\% 20$ general $\% 20 \mathrm{de} \% 201 \mathrm{la} \% 20 \mathrm{le}$ y\%20del\%20medio\%20ambiente.pdf

Organo Ejecutivo. El Salvador. (31 de Mayo de 2000). . Recuperado el 28 de Junio de 2014, de Reglamento Especial de Aguas Residuales:

HYPERLINK

"http://estadisticas.cne.gob.sv/images/boletines/Legislacion/ambiental/Reglamento _Aguas_Residuales.pdf"

http://estadisticas.cne.gob.sv/images/boletines/Legislacion/ambiental/Reglamento Aguas Residuales.pdf

Organo Ejecutivo. El Salvador. (31 de Mayo de 2000). Recuperado el 28 de Junio de 2014, de Reglamento Especial de Normas Técnicas de Calidad Ambiental: HYPERLINK

"http://estadisticas.cne.gob.sv/images/boletines/Legislacion/ambiental/Reglamento _Normas_Calidad_Ambiental.pdf"

http://estadisticas.cne.gob.sv/images/boletines/Legislacion/ambiental/Reglamento Normas Calidad Ambiental.pdf

Organo Ejecutivo. El Salvador. (31 de Mayo de 2000). Recuperado el 28 de Junio de 2014, de Reglamento Especial en Materia de Sustancias,Residuos y Desechos Peligrosos: $\quad$ HYPERLINK "http://faolex.fao.org/docs/pdf/els21262.pdf" http://faolex.fao.org/docs/pdf/els21262.pdf

Organo Ejecutivo. El Salvador. (31 de Mayo de 2000). Recuperado el 28 de Junio de 2014, de Reglamento Especial sobre el Control de las Sustancias Agotadoras de la Capa de Ozono: HYPERLINK "http://faolex.fao.org/docs/pdf/els21251.pdf" http://faolex.fao.org/docs/pdf/els21251.pdf

Ponce Talancón, H. (2007). La matriz FODA: Alternativa de diagnóstico y determinación de estrategias de intervención en diversas organizaciones. Enseñanza e investigación en psicología. 
Riechmann, J. (2006). Biomimesis: Ensayos sobre imitacion de la naturaleza, ecosocialismo y autocontención. Madrid: La Catarata.

Rodríguez, R. V. (2012). Cómo elaborar el análisis DAFO. Cuadernos Prácticos: Gestión Empresarial. Santiago de Compostela: CEEI Galicia, S.A.

SACDEL y CND. (2004). Plan de Desarrollo Turístico del circuito de la bahía de Jiquilisco y Sierra Tecapa Chinameca. Sistema de Asesoría y Capacitación para El Desarrollo Local, SACDEL.

Sáenz, R. M. (2010). Resumen Ambiental Nacional El Salvador. San Salvador: UNEP. United Nations Environment Programme.

Secretaría Técnica y de Planificación. (2015). Plan Quinquenal de Desarrollo 2014-2019. El Salvador productivo, educado y seguro. San Salvador: Presidencia de la República.

Sancho, E. (2011). Desarrollo Sostenible de la microrregión de Alegría: Municipios de Alegría, Mercedes Umaña, Jucuapa, Tecapán, Santiago de María. San Salvador: UFG Editores.

SENPLADES. Secretaría Nacional de Planificación y Desarrollo. Ecuador. (2012). Guía metodológica de planificación institucional. Quito: SENPLADES.

Silva, N., Macarrein, O., \& Forclaz, H. (2002). Desarrollo Económico y Medio Ambiente. XIII Reunión de Comunicaciones Científicas y Técnicas 2002. Facultad de Ciencias Agrarias UNNE.

UNESCO. (2012). Educación para el Desarrollo Sostenible. París: UNESCO.

Wilhelm, W. (1992). Changing corporate culture or corporate behavior? How to change your company. Academy of Management Executive, 72-76.

\section{Para citar este artículo:}

Mejía, R. (2016). Unificación de ordenanzas ambientales: Sierra Tecapa Chinameca, hacia la sostenibilidad local. Collectivus, Revista de Ciencias Sociales, 3(2), 85-106. doi: http://dx.doi.org/10.15648/Coll.2.2016.5 